\title{
Improving Quality in the Patients' Risk of Fall Evaluation through Clinical Supervision
}

\author{
Sandra Cruz, Luís Carvalho, Bárbara Lamas, and Pedro Barbosa
}

\begin{abstract}
In the hospital settings, falls occur for a wide scope of reasons but most of the time they could be prevented. Therefore, clinical supervision as a strategy to improve and develop nurses' knowledge and skills is crucial for a better clinical practice. The aims of this quantitative, exploratory and descriptive study were: to characterize the risk of falling of hospitalized patients in a unit of the medicine department; to relate the risk of falling with the patients' personal attributes; to analyze the compliance between the procedures for monitoring, planning and execution of nursing care; to suggest contributions for a clinical supervision model in nursing to improve nurses' skills in preventing falls. The population was composed by all the patients of the selected unit who were hospitalized from the 10th of February 2012 until the 9th of April 2012. We had a convenience sample constituted by 132 patients who fitted the inclusion criteria. We collected data through several procedures such as the application of a questionnaire to assess the risk of fall accordingly to the falls protocol implemented in the unit and the parameterization defined in the nursing records system. The results pointed out that $16,7 \%$ of patients showed "no risk of fall", $48,5 \%$ had "low risk of fall" and $34,8 \%$ had "high risk of fall". We verify the existence of non-conformities in the implementation of the falls protocol, the application of the Morse Fall Scale, among others. Thus, we recommend some contributions for a clinical supervision model in nursing so nurses can develop their knowledge and skills in preventing patients' falls.
\end{abstract}

Index Terms-Clinical supervision, risk of fall, patients' safety, quality of care.

\section{INTRODUCTION}

In recent decades, concern about safety and quality of health care has been a priority for all health professionals and institutions.

Patient safety should be a top priority in the improvement of health care and is one of the key dimensions of quality and risk management.

"There is a growing concern that the quality of hospital care needs to be improved and monitored systematically in many European Hospitals" [1].

The quality assessment is based on indicators that measure the performance of components of structure, process and outcome, comparing them to the desired standards.

The incidence of falls in a hospital setting is a care quality

Manuscript received November 3, 2013; revised January 13, 2014. Thi work was supported by Escola Superior de Enfermagem do Porto (ESEP) and Centro Hospitalar do Médio Ave EPE (CHMA).

Sandra Cruz, Luís Carvalho, and Barbara Lamas are with the Escola Superior de Enfermagem do Porto, 4200-072 Porto, Portugal (e-mail: sandracruz@esenf.pt, luiscarvalho@esenf.pt, ba_1928@hotmail.com).

Pedro Barbosa is with the Centro Hospitalar de São João, EPE, 4200-319 Porto, Portugal (e-mail: barbosapedro.ifm@gmail.com). indicator and the risk of falling is a nursing diagnosis operationalized through the application of scales. The scales are essential to assess the risk of falling because it help in the decision making, enables the appropriateness of interventions to each client, facilitates care planning and also the transmission of information between health professionals [2].

The early identification of a risk is a key aspect to injury prevention; however, this depends on the existence of a culture of trust, honesty, integrity and open communication between patients and nurses [3].

Some authors reported that factors such as the lack of balance and fear of falling are modifiable by doing exercises that include increase of strength and balance [4]. Nurses can provide exercises to reduce the number of falls [5], [6] but there is no clear evidence for identify the type, duration and / or intensity of exercise needed to the effective prevention [7].

Nurses are considered target professionals in the promotion and assurance of patient safety, especially in the prevention of falls. In their clinical practice, nurses, must use instruments to assess the risk of falling, identify risk factors and implement preventive measures. The most effective intervention for the prevention of fall in older adults is a multifactorial falls risk assessment and management program [8].

The implementation of a fall prevention plan requires a set of activities grounded in nurses' knowledge and skills. Therefore, clinical supervision as "a formal process of professional support and learning which enables individual practitioners to develop knowledge and competence, assume responsibility for their own practice and enhance consumer protection and safety of care in complex clinical situations" [9] is crucial to improve, develop and optimize nurses' knowledge and skills so, they can perform with excellence the care provided to their patients.

Thus, we decided to carry out a research placed within the paradigm of quantitative research to answer the following question: What are the aspects that should be included in a clinical supervision model in nursing to promote the safety and quality of care related to the risk of fall? This study took place in a unit of the medicine department of the Médio Ave Hospital Centre (CHMA) - Portugal.

With this article we intend to publicize the results of the first phase of an ongoing project titled: "Clinical Supervision for Safety and Care Quality" (C-S ${ }^{2}$ AFECARE-Q). This project results of a partnership from Nursing School of Porto (Escola Superior de Enfermagem do Porto - ESEP) and the Médio Ave Hospital Centre (Centro Hospitalar do Médio Ave - CHMA) and it is focused on the relation between clinical supervision, safety and the quality of nursing care.

This paper is divided into several main sections: the first 
one is related to the methodology and the study design; in the second one we present the results of the study and finally the discussion and the conclusion of it.

\section{Methodology And Study Design}

Clinical supervision is a formal process to monitor professional practice in order to develop the decision-making autonomy and, accordingly, to protect the patient, promoting the care safety through reflection and the analysis of clinical practices, which is hugely significant in a hospital context due to the complexity and unpredictability situations that professionals take care in this environment.

Nurses need continuous professional support in order to achieve better outcomes. Thus, we decided to carry out an exploratory and descriptive study placed within the paradigm of quantitative research to answer the following question: What are the aspects that should be included in a clinical supervision model in nursing to promote the safety and quality of care related to the risk of fall?

The aims of this study were: to characterize the risk of falling of hospitalized patients in a unit of the medicine department of the Médio Ave Hospital Centre (CHMA) Portugal; to relate the risk of falling with the patients' personal attributes; to analyze the compliance between the procedures for monitoring, planning and execution of nursing care; to suggest contributions for a clinical supervision model in nursing to improve nurses' skills in preventing falls.

Due to the starting question for this study and the aims defined we selected several research hypotheses such as:

H1-There is a statistically significant relationship between age and the risk of falling of the hospitalized patients in a unit of the medicine department of the CHMA.

$\mathrm{H} 2$-There is a statistically significant relationship between sex and the risk of falling of the hospitalized patients in a unit of the medicine department of the CHMA.

H3-There are statistically significant differences between the evaluation conducted by the researcher and what was documented in the nursing records at the time of patients' admission.

H4-There are statistically significant differences between the performed evaluation at the time of the patients' observation by the nurse responsible for the patient, the researcher and what was documented in the nursing records.

H5-There are statistically significant differences between the selected interventions by the nurse responsible for the patient and the interventions observed by the researcher and the documented ones in the nursing records.

H6-There are statistically significant differences between the selected nursing interventions by the nurse responsible for the patient about knowledge and learning skills to prevent falls and the interventions documented in the nursing records.

The population was composed by all the patients of the selected unit who were hospitalized from the $10^{\text {th }}$ of February 2012 until the $9^{\text {th }}$ of April 2012.

We had a convenience sample constituted by 132 patients who fulfilled the inclusion criteria which were: the patient should remain hospitalized for more than 24 hours, the patient should not have readmissions in the unit during the data collection and the patient should not have participated in the pretest of the questionnaire.

We collected data through several procedures performed in the same day, such as the application of a questionnaire to assess the risk of fall accordingly to the falls protocol implemented in the unit and the parameterization defined in the nursing records system.

Statistical Package for Social Sciences version 19.0 was used for data analysis.

The research took place in a unit of the medicine department of the Médio Ave Hospital Centre (CHMA) Portugal.

We obtained permission from the institution to conduct this research and the questionnaire had an introductory part where we explained the study and the ethical issues that we were going to respect like the anonymity and confidentiality of the collected information. We outlined the voluntary nature of the individuals' participation.

\section{RESULTS}

In our sample $(N=132)$, the mean of age was 72,5 years old with a standard deviation of 13 years. The younger patient had 25 years old and the older one had 97 years old.

The majority of the patients were male $(59,8 \% ; N=79)$. $47,0 \%(N=62)$ of the patients had an admission medical diagnosis of the group of Diseases and Disorders of the Respiratory System, 22,7\% $(N=30)$ of the patients had an admission medical diagnosis of the group of Diseases and Disorders of the Circulatory System and 9,8\% $(N=13)$ of the patients had an admission medical diagnosis of the group of Diseases and Disorders of the Kidney and Urinary System. The risk of fall was assessed by the researcher in the moment of patients' observation and the results were: 16,7\% $(N=22)$ of patients showed "no risk of fall", 48,5\% $(N=64)$ had "low risk of fall" and $34,8 \%(N=46)$ had "high risk of fall".

Appropriated statistical tests were used to assess the significant relations between the variables.

In Table I, we present the relation between the risk of fall and age (H1).

TABLE I: THE RISK OF FALL AND AGE

\begin{tabular}{llll}
\hline \hline $\begin{array}{l}\text { Age (years old) } \\
(N=132)\end{array}$ & $\begin{array}{l}\text { No Risk of } \\
\text { Fall }\end{array}$ & $\begin{array}{l}\text { Low Risk of } \\
\text { Fall }\end{array}$ & $\begin{array}{l}\text { High Risk of } \\
\text { Fall }\end{array}$ \\
\hline$\leq 40$ & $2(1,5 \%)$ & $1(0,8 \%)$ & $0(0,0 \%)$ \\
\hline $41-64$ & $11(8,3 \%)$ & $13(9,9 \%)$ & $7(5,3 \%)$ \\
\hline $65-79$ & $7(5,3 \%)$ & $26(19,7 \%)$ & $22(16,7 \%)$ \\
\hline$\geq 80$ years & $2(1,5 \%)$ & $24(18,2 \%)$ & $17(12,8 \%)$ \\
\hline \multicolumn{5}{c}{ Spearman Test $=0,264 ; N=132 ; p=0,002$} \\
\hline \hline
\end{tabular}

TABLE II: THE RISK OF FALL AND SEX

\begin{tabular}{llll}
\hline \hline Sex $(N=132)$ & $\begin{array}{l}\text { No Risk of } \\
\text { Fall }\end{array}$ & $\begin{array}{l}\text { Low Risk of } \\
\text { Fall }\end{array}$ & $\begin{array}{l}\text { High Risk of } \\
\text { Fall }\end{array}$ \\
\hline Male & $18(13,6 \%)$ & $37(28 \%)$ & $24(18,2 \%)$ \\
\hline Female & $4(3 \%)$ & $27(20,5 \%)$ & $22(16,7 \%)$ \\
\hline \multicolumn{2}{c}{ Kruskal Wallis Test ${ }^{(1)}(5,614) ;$ Significance level of $5 \%-P^{(1)} 0,060$} \\
\hline \hline
\end{tabular}

This data allow us to state that the risk of fall is influenced by the age of the patient. Age is a relevant factor in the risk of fall and should be taking into account by the health professionals. 
In Table II, we present the relation between the risk of fall and sex (H2).

From the analysis of Table II, we verified that there is no statistically significant relationship between sex and the risk of fall.

In our sample, the majority of patients $(85,6 \% ; N=113) \mathrm{did}$ not need any physical restraints and $14,4 \%(N=19)$ of the patients needed to be immobilized for their protection. From this 19 patients who needed to be immobilized, 36,8\% (N=7) did not fulfilled the falls protocol implemented in the institution.

In Table III, we present the relation between the risk of fall and the patients' physical restraint.

TABLE III: THE RISK OF FALL AND PATIENTS' PHYSICAL RESTRAINT

\begin{tabular}{llll}
\hline $\begin{array}{l}\text { Physical Restraint } \\
(\mathrm{N}=132)\end{array}$ & $\begin{array}{l}\text { No Risk of } \\
\text { Fall }\end{array}$ & $\begin{array}{l}\text { Low Risk of } \\
\text { Fall }\end{array}$ & $\begin{array}{l}\text { High Risk } \\
\text { of Fall }\end{array}$ \\
\hline $\begin{array}{l}\text { Yes } \\
(14,4 \% ; \mathrm{N}=19)\end{array}$ & $0(0 \%)$ & $\begin{array}{l}15(11,4 \%) / \\
(78,9 \%)\end{array}$ & $\begin{array}{l}4(3,03 \%) / \\
(21,1 \%)\end{array}$ \\
\hline $\begin{array}{l}\text { No } \\
(85,6 \% ; \mathrm{N}=113)\end{array}$ & $22(16,7 \%)$ & $49(37,1 \%)$ & $42(31,8 \%)$ \\
\hline \hline
\end{tabular}

From the analysis of Table III, we verified that from the 19 patients who needed physical restraint, $21,1 \%(N=4)$ had high risk of fall, 78,9\% $(N=15)$ had low risk of fall and did not fulfilled the falls protocol of the unit (only patients with high risk of falls should be immobilized).

In our sample, we verified that the procedure to assess the risk of fall at the moment of the patients' admission was correct for $33,3 \%(N=44)$ of the patients.

In Table IV, we present the relation between the evaluation conducted by the researcher and what was documented in the nursing records at the time of admission (H3).

TABLE IV: THE RISK OF FALL DOCUMENTED IN THE PATIENTS' ADMISSION AND THE ASSESSMENT BY THE RESEARCHER

\begin{tabular}{|c|c|c|c|}
\hline & $\begin{array}{l}\text { No Risk of } \\
\text { Fall }\end{array}$ & $\begin{array}{l}\text { Low Risk of } \\
\text { Fall }\end{array}$ & $\begin{array}{l}\text { High Risk } \\
\text { of Fall }\end{array}$ \\
\hline Documentation & $54(40,9 \%)$ & $61(46,2 \%)$ & $17(12,9 \%)$ \\
\hline Researcher & $21(15,9 \%)$ & $76(57,6 \%)$ & $35(26,5 \%)$ \\
\hline \multicolumn{4}{|c|}{ Mann Whitney Test ${ }^{(1)}(U=97,5)$; Significance level of $1 \% P<0,0001$} \\
\hline
\end{tabular}

From the analysis of Table IV, we verified that there are statistically significant differences between the evaluation conducted by the researcher and what was documented in the nursing records at the time of admission.

In Table $\mathrm{V}$, we present the relation between the performed evaluation at the time of the patients' observation by the nurse responsible for the patient, the researcher and what was documented in the nursing records (H4).

TABLE V: THE Risk OF FALl DOCUMENTED AT THE TIME OF PATIENTS' OBSERVATION AND THE ASSESSMENT BY THE NURSE RESPONSIBLE FOR THE PATIENT THE ASSESSMENT BY THE RESEARCHER

\begin{tabular}{llll} 
& $\begin{array}{l}\text { No Risk of } \\
\text { Fall }\end{array}$ & $\begin{array}{l}\text { Low Risk of } \\
\text { Fall }\end{array}$ & $\begin{array}{l}\text { High Risk } \\
\text { of Fall }\end{array}$ \\
\hline Documentation & $63(47,7 \%)$ & $56(42,4 \%)$ & $13(9,9 \%)$ \\
\hline Nurse & $58(43,9 \%)$ & $59(44,7 \%)$ & $15(11,4 \%)$ \\
\hline Researcher & $22(16,7 \%)$ & $64(48,5 \%)$ & $46(34,8 \%)$ \\
\hline
\end{tabular}

Kruskal Wallis Test H $(33,7), p<0,0001$; ${ }^{(2)}$ Mann Withney Test $U=12$; $p=0,42$ (documentation and nurse) $/{ }^{(3)} U=165 ; p<0,0001$ (documentation and researcher) $/{ }^{(4)} U=154 p<0,0001$ (nurse and researcher); Level of significance for all test $-1 \%$
From the analysis of Table $\mathrm{V}$, we verified that there are statistically significant differences between the evaluation conducted by the researcher and what was documented in the nursing records and the nurses' assessment.

In Table VI, we present the relation between the risk of fall and the nursing interventions (H5).

TABLE VI: THE RISK OF FALL AND THE NURSING INTERVENTIONS

\begin{tabular}{lll}
\hline \hline $\begin{array}{l}\text { Corrected Nursing } \\
\text { Interventions for the } \\
\text { Diagnosis }\end{array}$ & Yes & No \\
\hline Documentation & $54(40,9 \%)$ & $78(59,1 \%)$ \\
\hline Nurse & $65(49,2 \%)$ & $67(50,8 \%)$ \\
\hline Researcher & $59(44,7 \%)$ & $73(55,3 \%)$ \\
\hline${ }^{(1)}$ Kruskal Wallis Test H $(33,7) ; p<0,0001 ;{ }^{(2)}$ Mann Withney Test $U=$ \\
$\begin{array}{l}1353 ; p<0,0001{ }^{(3)} \text { Mann Withney Test } U=1370 ; p<0,0001 ;\left({ }^{4}\right) \text { Mann } \\
\text { Withney Test } U=1695 ; p<0,0001 ; \text { Level of significance for all test }-1 \%\end{array}$
\end{tabular}

From the analysis of Table VI, we verified that there are statistically significant differences between the selected interventions by the nurse responsible for the patient and the interventions observed by the researcher and the documented ones in the nursing records.

In Table VII, we present the relation the risk of fall and knowledge and learning skills (H6).

TABLE VII: THE RISK OF FALL AND KNOWLEDGE AND LEARNING SKILLS

\begin{tabular}{lcc}
\hline \hline $\begin{array}{l}\text { Corrected Nursing } \\
\text { Interventions for the } \\
\text { Diagnosis }\end{array}$ & Yes & No \\
\hline Documentation & $34(25,8 \%)$ & $98(74,2 \%)$ \\
\hline Nurse & $81(61,4 \%)$ & $51(38,6 \%)$ \\
\hline \multicolumn{2}{l}{ Mann Withney Test $U=1198 ;$ Significance level of $1 \% p<0,0001$} \\
\hline \hline
\end{tabular}

From the analysis of Table VII, we verified there are statistically significant differences between the selected nursing interventions by the nurse responsible for the patient about knowledge and learning skills to prevent falls and the interventions documented in the nursing records.

\section{DISCUSSION AND CONCLUSION}

In our sample, patients are predominantly males with over 65 years of age and with associated diseases.

The majority of patients $(n=110 ; 83,3 \%)$ presented risk of fall, while $16,7 \%(N=22)$ of the patients did not present any risk.

The World Health Organization Global Report on Falls Prevention in Older Age guide states that the risk of falling increases with age and weakness [10] and the National Patient Safety Agency refers that older people are more vulnerable to falls, and the patients who have fallen at least once are at increased risk of falling again [11].

The risk of accidental injury that results in death or hospitalization in older people is superior when compared to any other group [12].

This study highlights the need to implement a clinical supervision model to motivate, support and prepare nurses to optimize the fall prevention program and ensure the quality and safety of their patients.

Clinical supervision "can improve patient care, reduce errors, improve efficiency, enhance staff performance, and 
reduce burnout (Nicklin, 1997; Severinsson \& Borgenhammar, 1997). Other benefits to staff include improved job satisfaction, enhanced integration of theoretical and practical knowledge, and increase confidence, self-esteem, and empathy (Arvidsson, Löfgren \& Fridlund, 2001)" [13].

Falls in a hospital context is a phenomenon with an increasingly significant importance since it carries a set of physical consequences, psychological, social and economic negative impact on the patients' quality of life and their family.

Fall is a domain with relevance for clinical practice but also for clinical supervision because it is a quality indicator. Nurses can improve and develop their knowledge and skills in this area and provide excellence in nursing care to their patients through this results which can be an important subject for reflection in supervision.

Since the promotion of quality of care is one of the main clinical supervision assumptions, fall prevention is an area to explore and to pay attention in clinical supervision, for sure.

Regarding to the study hypotheses, we verified the existence of statistically significant differences between all hypotheses, except hypothesis 2 (fall risk / sex) and hypothesis 4 (assessment of the risk of falling by the responsible nurse / fall risk in the nursing records). We also verified non-conformities in the implementation of the institutional falls protocol, the application of physical restraint to patients and its relation to the risk of falling, among others.

According to the World Health Organization (2012), falls are the second leading cause of accidental death worldwide and are one of the major adverse events. Therefore, falls are a major challenge in the context of patients' security, quality of care [14] and in clinical supervision.

On the basis of our findings we suggest some contributions for a clinical supervision model. Those recommendations include: individual clinical supervision sessions to answer particular needs of each nurse, promote and develop their critical thinking, reflection, adaptability and flexibility; group clinical supervision sessions so the whole nursing team can present questions, share experiences and discuss clinical cases. Clinical supervision sessions have a huge importance in improving patient care because nurses can actively participate [15] and reflect on their practice. These strategies could lead the nursing team for further training and research, ensuring that nurses develop their clinical practice based on the best scientific evidence.

Due to the non-compliances identified in this study we are already conducting a research with aim to design a manual for the use of the Morse Fall Scale. This manual should be a tool to assist the nurses' daily practice in order to reduce the subjectivity in the interpretation of each item of the Morse Fall Scale promoting, by this way, accuracy in the assessment of the risk of fall and allowing the standardization in its implementation.

In nursing, "supervision is considered indispensable in the road for the quality of the teaching and of the practice" [16], it can "both empower and support those in practice but only if it is developed by clinical staff through them, as the process relies on those who are actively working in practice and have current experience. Clinical supervision involves a tripartite partnership between the supervisor, supervisee and the organization. If anyone of these fails to participate in, or actively sabotages clinical supervision, the supervisory process cannot be fully effective or demonstrably beneficial" [17].

It is essential to carry on further research on clinical supervision that highlights its contributions for the quality and safety of health care and also its positive effects on health professionals.

\section{ACKNOWLEDGMENT}

We gratefully acknowledge to the Nursing School of Porto (Escola Superior de Enfermagem do Porto - ESEP) and to the Médio Ave Hospital Centre (Centro Hospitalar do Médio Ave-CHMA) to allow this research and to collaborate with us.

\section{REFERENCES}

[1] W. Abreu and C. Marrow, "Clinical supervision in nursing practice: A comparative study in Portugal and in the United Kingdom," SANARE, vol. 11 , no. 2, pp. 16-24, 2012.

[2] D. Ganz et al., Preventing Falls in Hospitals - A Toolkit for Improving Quality of Care, Rockville: Agency for Healthcare Research and Quality, 2013.

[3] International Council of Nurses (ICN), Safe staffing saves lives. Information and Action Tool Kit, Switzerland: ICN, 2006.

[4] D. Skelton and N. Beyer, "Exercise and injury prevention in older people," Scan Journal of Medicine Science Sports, no. 13, pp. 77-85, 2003.

[5] M. Robertson et al., "Effectiveness and economic evaluation of a nurse delivered home exercise programme to prevent falls: One randomized controlled trial," British Medical Journal, no. 322, pp. 697-701, 2001.

[6] L. Day et al., "Randomized factorial trial of falls prevention among older people living in their own homes," British Medical Journal, vol. 325, no. 238, 2002.

[7] D. Rutledge, N. Donaldson, and D. Pravikoff, "Update 2003: fall risk assessment and prevention in hospitalized patients," Journal of Clinical Innovations, vol. 6, no. 5, pp. 1-55, Dec 2003.

[8] J. Chang et al., "Interventions for the prevention of falls in older adults: Systematic review and meta-analysis of randomized clinical trials," British Medical Journal, pp.328- 680, 2004.

[9] National Health Service, A Vision for the Future: The Nursing, Midwifery and Health Visiting Contribution to Health Care, London: Department of Health, 1993.

[10] World Health Organization (WHO), WHO Global Report on Falls Prevention in Older Age, France: WHO, 2007.

[11] National Patient Safety Agency, The Third Report from the Patient Safety Observatory: Slips, Trips and Falls in Hospital, London: NPSA, 2007.

[12] Registered Nurses Associations of Ontario (RNAO), Prevention of Falls and Fall Injuries in the Older Adult, Ontario: RNAO, 2005.

[13] M. Cleary and A. Freeman, "The cultural realities of clinical supervision in an acute inpatient mental health setting," Issues in Mental Health Nursing, vol. 26, pp. 489-505, 2005.

[14] World Health Organization (WHO). (2012). Falls. [Online]. Available: http://www.who.int/mediacentre/factsheets/fs344/en/

[15] A. Koivu, K. Hyrkas, and P. Saarinen, "Who attends clinical supervision? The uptake of clinical supervision by hospital nurses," Journal of Nursing Management, vol. 19, pp. 69-79, 2011.

[16] D. Sardo et al., Conditioning Factors in Nursing Students Clinical Supervision, In Recent Research Developments in Learning Technologies, Badajoz: Formatex, vol. 1, pp. 402-405, 2005.

[17] National Health Service, Clinical Supervision Policy, Leicestershire County and Rutland: National Health Service Primary Care Trust, 2006.

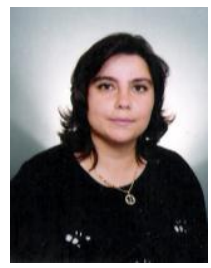

Sandra Cruz was born in Porto-Portugal, 1972. She earned her doctoral degree from the Catholic University of Portugal and her master degree in administration and planning education from the Portucalense University-Portugal. Her doctoral research was on clinical supervision in nursing and she designed and developed the 'Contextualized Clinical Supervision Model in Nursing'. 
She is an associate professor at the Nursing School of Porto - Portugal (ESEP) and recently became a member of the ESEP Pedagogical Board. She has been an Invited Professor (of clinical supervision) in several Portuguese Institutions such as the Catholic University of Portugal; CESPU Cooperative Higher Education, Polytechnic and University and at the IPVC Polytechnic Institute of Viana do Castelo.

Professor Cruz is a member of the Portuguese Nurses Order since 1998. She is a member of the ESEP Research Unit (UNIESEP) and recently she became a member of the Center for Research in Health Technologies and Health Services (CINTESIS).

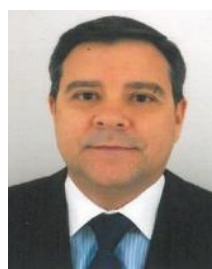

Luís Carvalho was born in 1963. He earned the PhD in education sciences in the University of Santiago de Compostela - Spain (2003) and the master in nursing sciences in the University of Porto (1996).

He is a coordinator professor at the Nursing School of Porto since 1989 where he is the president of the Technical and Scientific Council. He is a member of the ESEP Research Unit (UNIESEP) and Coordinator of the research project "Clinical Supervision for Safety and Care Quality" (C-S ${ }^{2}$ AFECARE-Q).

Professor Carvalho is the Regent of the following disciplines: Health Information and Education in Clinical Context. He develops also teaching activities in the PhD in Education at the Lusófona University of Porto. He collaborates as advisor and examiner of several master and doctorate thesis in nursing and education.

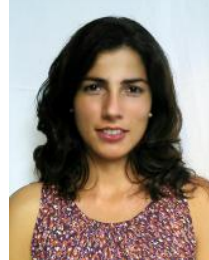

Bárbara Lamas was born in Porto-Portugal, 1986. She earned her master degree in clinical supervision in Nursing from Nursing School of Porto (ESEP)-Portugal in 2012.

She worked as a registered nurse at the Portuguese Institute of Oncology-Porto until April 2013. Currently, she is an invited assistant at the Nursing School of Porto-Portugal.

Bárbara Lamas has been member of the Portuguese Nurses Order since 2009.

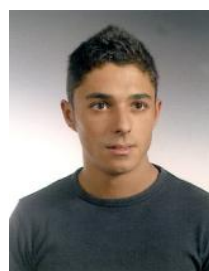

Pedro Barbosa born in Valongo-Portugal, 1986. He earned his nursing degree in the Nursing School of Porto in 2009, his Master's Clinical Supervision in Nursing in November 2013 at the Nursing School of Porto

He works as a registered nurse in the Neurology Department of the São João Hospital Centre EPE-Porto since 2010 .

Pedro Barbosa has been member of the Portuguese Nurses Order since 2009 